\title{
Correlation and Path Analysis Study in Studied in Ridge Gourd (Luffa acutangula (L.) Roxb.)
}

\author{
N.D. Ramesh ${ }^{1 *}$, Praveen Choyal ${ }^{1}$, Radhelal Dewangan ${ }^{1}$, Pushpa S. Gudadinni ${ }^{1}$, \\ Priyanka P. Ligade ${ }^{1}$ and Kalyan Singh Seervi ${ }^{2}$ \\ ${ }^{1}$ Department of Horticulture, SHUATS, Allahabad (U.P.), India \\ ${ }^{2}$ Department of Genetics and Plant Breeding, SHUATS, Allahabad (U.P.), India \\ *Corresponding author
}

\section{A B S T R A C T}

\begin{tabular}{|c|}
\hline Keywords \\
\hline $\begin{array}{l}\text { Ridge gourd ( } L u f f a \\
\text { acutangula (L.) Roxb.), } \\
\text { Fruit yield, Correlation } \\
\text { and path analysis }\end{array}$ \\
\hline Article Info \\
\hline $\begin{array}{l}\text { Accepted: } \\
\text { 10 July } 2018 \\
\text { Available Online: } \\
\text { 10 August } 2018\end{array}$ \\
\hline
\end{tabular}

In the present investigation, 14 genotypes will be grown during rainy season 2016-2017 at the field experimentation centre of the Department of Horticulture, SHUATS, Allahabad (U.P.). The overall analysis reveals that fruit yield was positively and significant correlated with fruit percent, number of fruits per plant, fruit length, average fruit weight, fruit diameter, vine length at 90 days, sex ratio and rind thickness while selecting a good hybrid for enhancing the yield of ridge gourd. In ridge gourd 16 important growth, earliness, yield and quality parameters were subjected to Genotypic and Phenotypic path coefficient analysis. Fruit yield per plot had high positive direct effect on yield. Positive indirect effect was observed through node to first male flowering, days to last harvest, number of fruits per plant, average fruit weight, fruit length and fruit diameter. Negative indirect effect were observed through vine length 90 days after sowing, days to first female flowering, days to first male flowering, node to first female flowering, days to 50 per cent flowering, days to first harvest, and sex ratio.

\section{Introduction}

The ridge gourd belongs to the cucurbitaceous family. It is also known as a ribbed gourd. This vegetable is not so commonly grown in all over the world. But, the demand for this vegetable is slowly increasing and hence cultivation for this vegetable has become important in the coming years. The genus derives its name from the product "loofah" which is used in bathing sponges, scrubber pads, door mats, pillows, mattresses and also for cleaning utensils. India has the credibility of producing 169.478 million tonnes of vegetables covering an area of 9.542 million hectares in 2014-15 (NHB, 2014-15) securing the second status among the vegetable producing countries of the world but the per capita availability of vegetables in India is as low as $160 \mathrm{gm}$ as against the recommended $300 \mathrm{~g}$ per day by FAO. Association of characters determined by correlation coefficient, although useful will not provide an exact picture of the relative importance of direct and indirect influence of each of the characters towards yield. Path co-efficient analysis was developed by Wright (1921) has been employed in many vegetables in order to 
overcome the unreliability of correlation coefficient, this technique involves effective partitioning of the correlation co-efficient in to measures of direct and indirect effects on yield.

\section{Materials and Methods}

In the present investigation fourteen genotypes of ridge guard was grown in a randomized block design with three replications. The varieties were grown in a randomized block design with three replicates, keep row to row distance of $1.2 \mathrm{~m}$. and plant to plant distance of $0.90 \mathrm{~m}$.

Observation were recorded on five randomly selected plants per treatment for eighteen quantitative characters viz., days to taken 1st Male Flowering, days to taken 1st female flowering, days to $50 \%$ Flowering, node to first male flower, node to first female flower Sex Ratio, vine length $\mathrm{cm}$ at 90 days, days to 1st harvest, days to last harvest, fruit length, flesh thickness, rind thickness, fruit set \%, fruit diameter, fruits/ Plant, average fruit weight, fruit yield/ plant, fruit yield.

\section{Results and Discussion}

The results of the analysis of variance for different quantitative characters for 14 genotypes of ridge gourd are presented in (Table 1). Analysis of variance presented in showed that the genotypes differed significantly for all the 18 characters and the mean performance of 14 genotypes.

As correlated coefficients are the index of association between two variables; these have been worked out in all possible combinations at genotypic $(\mathrm{G})$ and phenotypic $(\mathrm{P})$ levels.

Days to first male flowering had highly significant and positively associated with days to $50 \%$ flowering $(0.966)$ days to taken first female flower (0.962), and days to 1st harvest (0.565), node to first female flower (0.564), vine length at 90 Days (0.403).

Days to first female flowering was highly significant and positively associated with days to 50 per cent flowering (0.97), days to first harvest $(0.756)$, node to first female flower (0.675), node to first male flower (0.479).

Days to 50 per cent flowering had highly significant and positive association with days to first harvest (0.739), node to first female flower (0.608), days to last harvest (0.437), node to first male flower $(0.385)$.

Node to first male flowering had highly significant and positive association with node to first female flower (0.833), days to first harvest (0.526), days to last harvest (0.332).

Node to first female flowering had highly significant and positive correlation with days to first harvest (0.589).

Sex ratio was highly significant and positive association with vine length at 90 days (0.774), average fruit weight (0.694), fruit yield per plant (0.590), fruit length (0.546), per cent fruit set (0.468), number of fruits per plant (0.461), and fruit diameter (0.422).

Vine length at 90 days after sowing was highly significant and positively associated with fruit length (0.779), average fruit weight (0.714), fruit yield per plant (0.624), fruit set percent (0.538), number of fruits per plant $(0.517)$ ), fruit diameter $(0.479)$, rind thickness (0.43), flesh thickness (0.362).

Days to first harvest had highly significant and negative association with fruit set percent ($0.379)$, fruits per plant (-0.457), also positive and non-significant with days to last harvest (0.369), fruits diameter (0.053), average fruit weight (0.048) (Table 2). 
Table.1a Genotypic correlation coefficients among growth, earliness, yield and yield components in ridge gourd

\begin{tabular}{|c|c|c|c|c|c|c|c|c|c|c|c|c|c|c|c|c|c|c|c|}
\hline Chr. & 1 & 2 & 3 & 4 & 5 & 6 & 7 & 8 & 9 & 10 & 11 & 12 & 13 & 14 & 15 & 16 & 17 & 18 & 19 \\
\hline 1 & 1.00 & $0.962 * *$ & $0.966 * *$ & 0.314 & $0.564 * *$ & 0.188 & $0.403 *$ & $0.565^{* *}$ & 0.228 & 0.195 & 0.112 & $\overline{-} .2010$ & -0.0870 & 0.0348 & -0.1900 & 0.2120 & -0.0930 & -0.0946 & -0.0947 \\
\hline 2 & & 1.0000 & $0.97 * *$ & $0.479 * *$ & $0.675^{* *}$ & 0.122 & 0.2341 & $0.756^{* *}$ & 0.2461 & 0.0991 & -0.006 & -0.294 & -0.297 & -0.005 & $-0.388^{*}$ & 0.1537 & -0.247 & -0.246 & -0.248 \\
\hline 3 & & & 1.0000 & $0.384 *$ & $0.608^{* *}$ & 0.106 & 0.271 & $0.739^{* *}$ & $0.437 *$ & 0.155 & -0.0836 & $\overline{-} .2025$ & -0.1917 & -0.0259 & -0.2671 & 0.124 & -0.1768 & -0.1745 & -0.1730 \\
\hline 4 & & & & 1.0000 & $0.833 * *$ & 0.010 & -0.0129 & $0.5269 * *$ & $0.3325^{*}$ & -0.0563 & $\overline{0.4283 * *}$ & 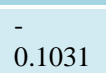 & $\overline{0.569 * *}$ & -0.0754 & $\overline{-}^{0.516^{* *}}$ & 0.0995 & -0.3406 & -0.3380 & $-0.37 *$ \\
\hline 5 & & & & & 1.0000 & 0.032 & -0.0033 & $0.589^{* *}$ & 0.2843 & -0.2644 & $-0.352^{*}$ & $\overline{-} .2765$ & $\overline{-}-572 * *$ & -0.2599 & $-\overline{0.596 * *}$ & 0.0152 & $\overline{-}-444 *$ & $\overline{-}-449 * *$ & $-0.447 * *$ \\
\hline 6 & & & & & & 1.0000 & $0.7742 * *$ & -0.1666 & 0.0357 & 0.5463 & 0.1271 & 0.2648 & $0.468^{* *}$ & $0.422 * *$ & $0.461^{* *}$ & $0.694 * *$ & $0.590^{* *}$ & $0.589^{* *}$ & $0.593 * *$ \\
\hline 7 & & & & & & & 1.0000 & -0.1918 & 0.1161 & $0.7793 * *$ & $0.362 *$ & $0.43 * *$ & $0.538^{* * *}$ & 0.479 ** & $0.517 * *$ & $0.714^{* *}$ & $0.624 * *$ & 0.621 ** & $0.622 * *$ \\
\hline 8 & & & & & & & & 1.0000 & 0.3694 & -0.0255 & -0.2612 & 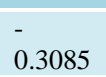 & $\overline{-}-379 * *$ & 0.0539 & $\overline{0.457 * *}$ & 0.0480 & -0.2907 & -0.2865 & -0.2892 \\
\hline 9 & & & & & & & & & 1.0000 & 0.2413 & $-0.392 *$ & $0.47 * *$ & -0.0237 & 0.0092 & 0.0583 & -0.0158 & 0.0413 & 0.0360 & 0.0330 \\
\hline 10 & & & & & & & & & & 1.0000 & $0.446^{* *}$ & $0.68^{* *}$ & $0.657^{* *}$ & $0.849 * *$ & $0.629 * *$ & $0.828^{* *}$ & $0.812^{* *}$ & $0.805^{* *}$ & $0.809^{* *}$ \\
\hline 11 & & & & & & & & & & & 1.0000 & $0.45^{* *}$ & 0.276 & $0.498^{* *}$ & 0.165 & $0.348^{*}$ & 0.2619 & 0.2620 & 0.2625 \\
\hline 12 & & & & & & & & & & & & 1.0000 & $0.444 * *$ & $0.561^{* *}$ & $0.481 * *$ & $0.416^{* *}$ & $0.535^{* *}$ & $0.531^{* *}$ & $0.534 * *$ \\
\hline 13 & & & & & & & & & & & & & 1.0000 & $0.531 * *$ & $0.999 * *$ & $0.529 * *$ & $0.927 * *$ & $0.918 * *$ & $0.923 * *$ \\
\hline 14 & & & & & & & & & & & & & & 1.0000 & $0.495^{* *}$ & $0.876 * *$ & 0.763 ** & $0.762 * *$ & $0.766^{* *}$ \\
\hline 15 & & & & & & & & & & & & & & & 1.0000 & $0.493 * *$ & $0.920^{* *}$ & 0.919 ** & $0.925 * *$ \\
\hline 16 & & & & & & & & & & & & & & & & 1.0000 & $0.800^{* *}$ & $0.796^{* *}$ & $0.800 * *$ \\
\hline 17 & & & & & & & & & & & & & & & & & 1.0000 & $1.007^{* *}$ & $1.011 * *$ \\
\hline 18 & & & & & & & & & & & & & & & & & & 1.0000 & $1.008 * *$ \\
\hline 19 & & & & & & & & & & & & & & & & & & & 1.0000 \\
\hline
\end{tabular}

1 Days to Taken 1st Male Flowering 2. Days to Taken 1st Female Flowering 3. Days to 50\% Flowering 4. Node to First Male Flower 5. Node to First Female Flower 6.Sex Ratio 7.Vine Length $\mathrm{cm}$ At 90 Days 8.Days to 1st harvest 9.Days to Last harvest 10.Fruit Length $\mathrm{cm}$ 11.Flesh Thickness $\mathrm{cm}$ 12.Rind Thickness $\mathrm{mm}$ 13.Fruit Set \% 14.Fruit Diameter 15.Fruits/ Plant 16.Average Fruit Weight (g) 17.Fruit Yield/ Plant (g) 18.Fruit Yield/ Plot (kg) 19.Fruit Yield Q/ha 
Table.1b Phenotypic correlation coefficients among growth, earliness, yield and yield components in ridge gourd

\begin{tabular}{|c|c|c|c|c|c|c|c|c|c|c|c|c|c|c|c|c|c|c|c|}
\hline Chr. & 1 & 2 & 3 & 4 & 5 & 6 & 7 & 8 & 9 & 10 & 11 & 12 & 13 & 14 & 15 & 16 & 17 & 18 & 19 \\
\hline 1 & 1.0000 & $0.935^{* *}$ & $\begin{array}{l}0.905 \\
* *\end{array}$ & 0.2818 & $0.5469 * *$ & 0.1812 & $0.390 *$ & $0.536^{* *}$ & 0.1674 & 0.1975 & 0.0929 & -0.187 & -0.082 & 0.033 & -0.176 & 0.204 & -0.099 & -0.096 & -0.096 \\
\hline 2 & & 1.00 & $\begin{array}{l}0.924 \\
* *\end{array}$ & $0.455^{* *}$ & $0.654 * *$ & 0.1243 & 0.2255 & $0.713 * *$ & 0.2113 & 0.0845 & -0.0228 & -0.268 & -0.258 & -0.002 & $-0.363 *$ & 0.152 & -0.235 & -0.238 & -0.235 \\
\hline 3 & & & 1.00 & $0.3571^{*}$ & $0.5882 * *$ & 0.1039 & 0.2665 & $0.6726^{* *}$ & $0.3853^{*}$ & 0.1373 & -0.0902 & -0.195 & -0.177 & -0.031 & -0.251 & 0.106 & -0.160 & -0.165 & -0.168 \\
\hline 4 & & & & 1.0000 & $0.7704 * *$ & 0.0185 & -0.0322 & $0.4729 * *$ & $0.3138^{*}$ & -0.0679 & $-0.411 * *$ & -0.089 & $\overline{0} .527 * *$ & -0.062 & $\overline{-}-472 * *$ & 0.092 & -0.299 & -0.304 & $-0.306 *$ \\
\hline 5 & & & & & 1.0000 & 0.0267 & 0.0100 & $0.5386^{* *}$ & 0.2354 & -0.2576 & $-0.338^{*}$ & -0.262 & $\overline{0} .536 * *$ & -0.266 & $\overline{-}-584 * *$ & 0.024 & $\overline{-}-443 * *$ & $\overline{0}-434 * *$ & $\overline{-} \mathbf{5} 437 * *$ \\
\hline 6 & & & & & & 1.0000 & $0.7495 * *$ & -0.1477 & 0.0404 & $0.5246^{* *}$ & 0.1174 & 0.259 & $0.434 * *$ & $0.415 * *$ & $0.454 * *$ & $0.682 * *$ & $0.580 * *$ & $0.582 * *$ & $0.57 * *$ \\
\hline 7 & & & & & & & 1.0000 & -0.1868 & 0.0881 & $0.7473 * *$ & $0.349 *$ & $0.411 * *$ & $0.519 * *$ & $0.461 * *$ & $0.482 * *$ & 0.690 ** & $0.596 * *$ & $0.602 * *$ & $0.600 * *$ \\
\hline 8 & & & & & & & & 1.0000 & 0.2999 & -0.0163 & -0.2568 & -0.297 & $-0.328 *$ & 0.041 & $\overline{-}-413 * *$ & 0.050 & -0.261 & -0.269 & -0.264 \\
\hline 9 & & & & & & & & & 1.0000 & 0.2106 & $-0.334 *$ & $0.411 * *$ & -0.007 & 0.024 & 0.061 & 0.010 & 0.040 & 0.050 & 0.055 \\
\hline 10 & & & & & & & & & & 1.00 & $0.429 * *$ & $0.647 * *$ & $0.590 * *$ & $0.829 * *$ & $0.607 * *$ & $0.793 * *$ & $0.769 * *$ & $0.781 * *$ & $0.775 * *$ \\
\hline 11 & & & & & & & & & & & 1.00 & $0.436 * *$ & 0.254 & $0.474 * *$ & 0.148 & $0.310 *$ & 0.247 & 0.247 & 0.246 \\
\hline 12 & & & & & & & & & & & & 1.00 & $0.437 * *$ & $0.546 * *$ & $0.458 * *$ & $0.402 * *$ & $0.508 * *$ & $0.517 * *$ & $0.511 * *$ \\
\hline 13 & & & & & & & & & & & & & 1.00 & $0.496 * *$ & $0.925 * *$ & $0.500 * *$ & $0.841 * *$ & $0.857 * *$ & $0.849 * *$ \\
\hline 14 & & & & & & & & & & & & & & 1.00 & $0.477 * *$ & $0.849 * *$ & $0.751 * *$ & $0.754 * *$ & $0.745 * *$ \\
\hline 15 & & & & $*$ & & & & & & & & & & & 1.00 & $0.472 * *$ & $0.878 * *$ & $0.891 * *$ & $0.880 * *$ \\
\hline 16 & & & & & & & & & & & & & & & & 1.00 & $0.770 * *$ & $0.777 * *$ & $0.768 * *$ \\
\hline 17 & & & & & & & & & & & & & & & & & 1.00 & $0.986 * *$ & $0.978 * *$ \\
\hline 18 & & & & & & & & & & & & & & & & & & 1.00 & $0.983 * *$ \\
\hline 19 & & & & & & & & $*$ & & & & & & & & & & & 1.00 \\
\hline
\end{tabular}

1 Days to Taken 1st Male Flowering 2. Days to Taken 1st Female Flowering 3. Days to 50\% Flowering 4. Node to First Male Flower 5. Node to First Female Flower 6.Sex Ratio 7.Vine Length $\mathrm{cm}$ At 90 Days 8.Days to 1st harvest 9.Days to Last harvest 10.Fruit Length $\mathrm{cm} 11$. Flesh Thickness $\mathrm{cm} \mathrm{12.Rind} \mathrm{Thickness}$ $\mathrm{mm}$ 13.Fruit Set \% 14.Fruit Diameter 15.Fruits/ Plant 16.Average Fruit Weight (g) 17.Fruit Yield/ Plant (g) 18.Fruit Yield/ Plot (kg) 19.Fruit Yield Q/ha 
Table.2a Genotypic path coefficient analysis among growth, earliness, yield and yield component in ridge gourd

\begin{tabular}{|c|c|c|c|c|c|c|c|c|c|c|c|c|c|c|c|c|}
\hline Character & 1 & 2 & 3 & 4 & 5 & 6 & 7 & 8 & 9 & 10 & 11 & 12 & 13 & 14 & 15 & 16 \\
\hline 1 & -0.2239 & -0.2154 & -0.2163 & -0.0703 & -0.1262 & -0.0422 & -0.0903 & -0.1267 & -0.0511 & -0.0438 & -0.0252 & 0.0450 & 0.0195 & -0.0078 & 0.0425 & -0.0475 \\
\hline 2 & -0.3975 & -0.4131 & -0.4011 & -0.1979 & -0.2792 & -0.0507 & -0.0967 & -0.3124 & -0.1017 & -0.0409 & 0.0024 & 0.1214 & 0.1228 & 0.0023 & 0.1603 & -0.0635 \\
\hline 3 & 0.6507 & 0.6537 & 0.6733 & 0.2590 & 0.4099 & 0.0718 & 0.1825 & 0.4976 & 0.2946 & 0.1047 & -0.0563 & -0.1363 & -0.1291 & -0.0175 & -0.1798 & 0.0836 \\
\hline 4 & -0.0967 & -0.1475 & -0.1184 & -0.3078 & -0.2567 & -0.0032 & 0.0040 & -0.1622 & -0.1024 & 0.0173 & 0.1319 & 0.0317 & 0.1752 & 0.0232 & 0.1589 & -0.0306 \\
\hline 5 & 0.2856 & 0.3423 & 0.3083 & 0.4223 & 0.5065 & 0.0167 & -0.0017 & 0.2985 & 0.1440 & -0.1339 & -0.1786 & -0.1400 & -0.2902 & -0.1316 & -0.3018 & 0.0077 \\
\hline 6 & 0.0210 & 0.0137 & 0.0119 & 0.0012 & 0.0037 & 0.1114 & 0.0862 & -0.0186 & 0.0040 & 0.0608 & 0.0142 & 0.0295 & 0.0522 & 0.0470 & 0.0514 & 0.0774 \\
\hline 7 & -0.1800 & -0.1045 & -0.1210 & 0.0057 & 0.0015 & -0.3455 & -0.4463 & 0.0856 & -0.0518 & -0.3478 & -0.1617 & -0.1908 & -0.2405 & -0.2139 & -0.2310 & -0.3190 \\
\hline 8 & -0.1828 & -0.2443 & -0.2387 & -0.1702 & -0.1904 & 0.0538 & 0.0620 & -0.3230 & -0.1193 & 0.0082 & 0.0844 & 0.0997 & 0.1226 & -0.0174 & 0.1477 & -0.0155 \\
\hline 9 & -0.0821 & -0.0885 & -0.1574 & -0.1196 & -0.1023 & -0.0129 & -0.0418 & -0.1329 & -0.3597 & -0.0868 & 0.1411 & -0.1690 & 0.0085 & -0.0033 & -0.0210 & 0.0057 \\
\hline 10 & 0.1690 & 0.0855 & 0.1341 & -0.0486 & -0.2282 & 0.4714 & 0.6725 & -0.0220 & 0.2083 & 0.8630 & 0.3850 & 0.5898 & 0.5675 & 0.7332 & 0.5434 & 0.7148 \\
\hline 11 & -0.0431 & 0.0022 & 0.0320 & 0.1639 & 0.1349 & -0.0486 & -0.1386 & 0.0999 & 0.1501 & -0.1707 & -0.3826 & -0.1745 & -0.1059 & -0.1909 & -0.0634 & -0.1281 \\
\hline 12 & -0.0240 & -0.0351 & -0.0242 & -0.0123 & -0.0330 & 0.0316 & 0.0511 & -0.0368 & 0.0561 & 0.0816 & 0.0545 & 0.1194 & 0.0531 & 0.0671 & 0.0575 & 0.0497 \\
\hline 13 & -0.0588 & -0.2009 & -0.1295 & -0.3847 & -0.3873 & 0.3167 & 0.3642 & -0.2566 & -0.0160 & 0.4444 & 0.1870 & 0.3007 & 0.6759 & 0.3593 & 0.6756 & 0.3577 \\
\hline 14 & 0.0051 & -0.0008 & -0.0038 & -0.0110 & -0.0379 & 0.0615 & 0.0699 & 0.0079 & 0.0013 & 0.1239 & 0.0728 & 0.0819 & 0.0775 & 0.1458 & 0.0722 & 0.1278 \\
\hline 15 & 0.0443 & 0.0904 & 0.0622 & 0.1202 & 0.1388 & -0.1074 & -0.1205 & 0.1064 & -0.0136 & -0.1466 & -0.0386 & -0.1122 & -0.2328 & -0.1154 & -0.2329 & -0.1150 \\
\hline 16 & 0.0202 & 0.0146 & 0.0118 & 0.0095 & 0.0014 & 0.0662 & 0.0680 & 0.0046 & -0.0015 & 0.0788 & 0.0319 & 0.0396 & 0.0504 & 0.0834 & 0.0470 & 0.0952 \\
\hline 17 & -0.0930 & -0.2476 & -0.1768 & -0.3406 & -0.4446 & 0.5907 & 0.6245 & -0.2907 & 0.0413 & 0.8124 & 0.2619 & 0.5359 & 0.9270 & 0.7635 & 0.9266 & 0.8004 \\
\hline Partial $\mathbf{R}^{2}$ & 0.0208 & 0.1023 & -0.1190 & 0.1049 & -0.2252 & 0.0658 & -0.2787 & 0.0939 & -0.0149 & 0.7011 & -0.1002 & 0.0640 & 0.6265 & 0.1113 & -0.2158 & 0.0762 \\
\hline
\end{tabular}

1 Days to Taken 1st Male Flowering 2. Days to Taken 1st Female Flowering 3. Days to 50\% Flowering 4. Node to First Male Flower 5. Node to First Female Flower 6.Sex Ratio 7.Vine Length $\mathrm{cm}$ At 90 Days 8.Days to 1st harvest 9.Days to Last harvest 10.Fruit Length $\mathrm{cm}$ 11.Flesh Thickness $\mathrm{cm}$ 12.Rind Thickness $\mathrm{mm}$ 13.Fruit Set \% 14.Fruit Diameter 15.Fruits/ Plant 16.Average Fruit Weight (g) 17.Fruit Yield/ Plant (g) 18.Fruit Yield/ Plot (kg) 19.Fruit Yield Q/ha 
Table.2b Phenotypic path coefficient analysis among growth, earliness, yield and yield component in ridge gourd

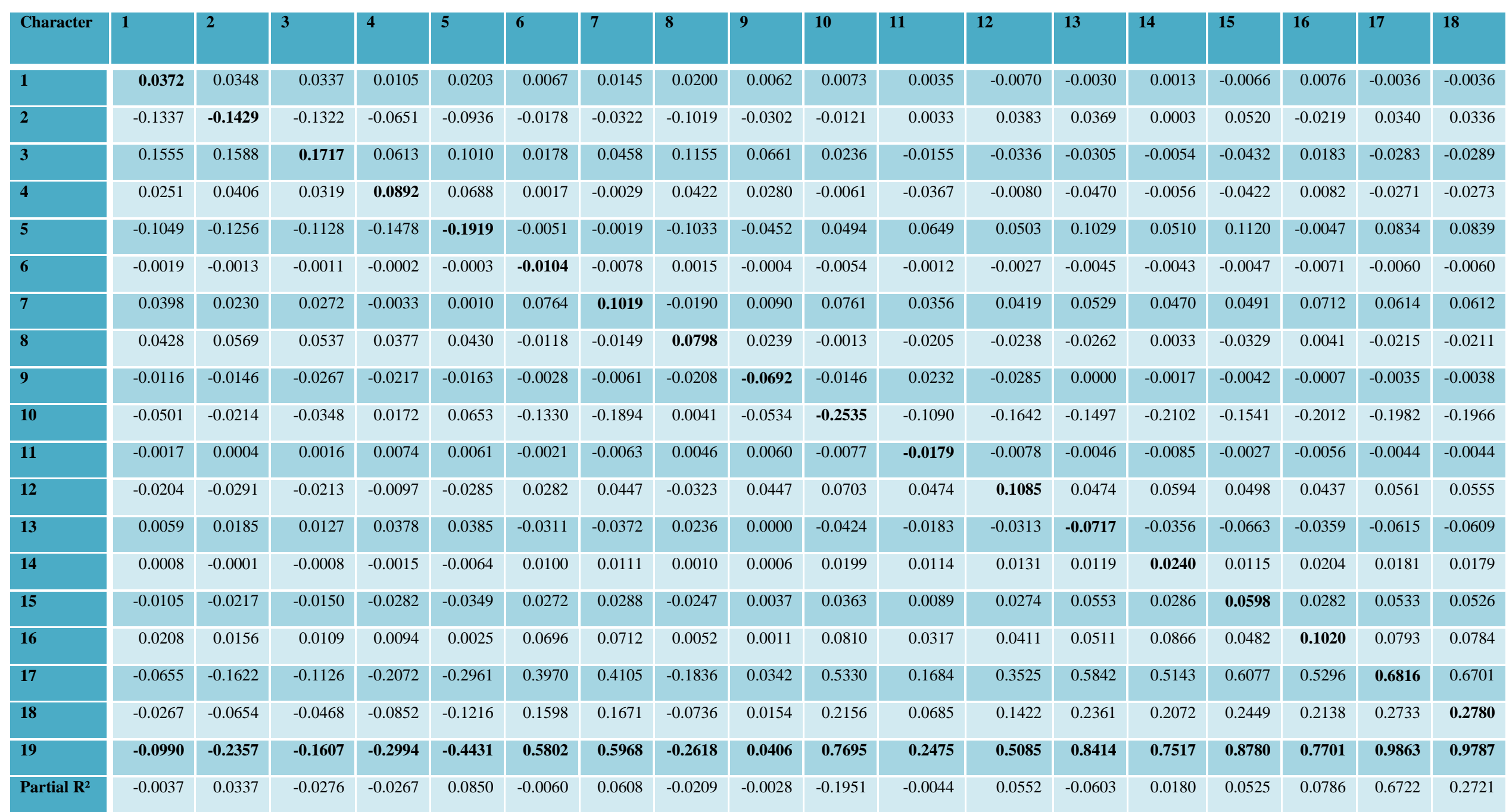

1 Days to Taken 1st Male Flowering 2. Days to Taken 1st Female Flowering 3. Days to 50\% Flowering 4. Node to First Male Flower 5. Node to First Female Flower 6.Sex Ratio 7.Vine Length $\mathrm{cm}$ At 90 Days 8.Days to 1st harvest 9.Days to Last harvest 10.Fruit Length $\mathrm{cm}$ 11.Flesh Thickness $\mathrm{cm}$ 12.Rind Thickness $\mathrm{mm}$ 13.Fruit Set \% 14.Fruit Diameter 15.Fruits/ Plant 16.Average Fruit Weight (g) 17.Fruit Yield/ Plant (g) 18.Fruit Yield/ Plot (kg) 19.Fruit Yield Q/ha 
Days to first harvest had positive and nonsignificant association with days to last harvest (0.369), fruit diameter (0.0539) and average fruit weight (0.0480).

Days to last harvest had highly significant and positive association with rind thickness (0.47).

Fruit length $\mathrm{cm}$ had highly significant and positive association with fruit diameter (0.849), average fruit weight (0.828), fruit yield per plant (0.812), rind thickness (0.68), fruit percent $(0.657)$, number of fruits per plant (0.629), rind thickness (0.446), flesh thickness.

Rind thickness $\mathrm{cm}$ was highly significant and positively associated with fruit diameter $\mathrm{cm}$ (0.561), fruit yield per plant (0.535), number of fruits per plant (0.481), fruit set percent (0.444), average fruit weight (0.416). fruit Set percent was highly significant and positively associated with number of fruits per plant (0.999), fruit yield per plant (0.927), fruit diameter (0.531), average fruit weight (0.529).

Fruit diameter was highly significant and positively associated with average fruit weight (0.876), fruit yield per plant (0.763), number of fruits per plant $(0.495)$.

Number of fruits per plant was highly significant and positively associated with fruit yield per plant (0.920), average fruit weight (0.493). Similar results were obtained by Srivastava and Srivastava (1976), Mangal et al., (1981), Khattra et al., (1994) and Rajeswari (1998).

Average fruit weight was highly significant and positively associated with fruit yield per plant (0.800).

Fruits yield per plant $\mathrm{kg}$ was highly significant and positively associated with fruit percent (0.927), number of fruits per plant (0.920), fruit length (0.812), average fruit weight (0.800), fruit diameter (0.763), vine length at 90 days $(0.624)$, sex ratio $(0.590)$, rind thickness (0.535). These results are in confirmed with Varalakshmi et al., (1995), Rao et al., (2000), Chowdhury and Sarma (2002) and Prasanna et al., (2002) in ridge gourd.

\section{Path analysis}

Days to first male flower had direct negative effect (-0.2239) on yield. Negative indirect effect were seen through vine length 90 days after sowing (-0.0903), days to first female flowering (-0.2154), node to first female flowering (-0.1262), days to 50 per cent flowering (-0.2163), days to first harvest ($0.1267)$, sex ratio (-0.042), Days to Last harvest (-0.051), Fruit Length $\mathrm{cm}(-0.044)$, Flesh Thickness cm (-0.0252), Fruit Diameter $(-0.008)$ and Average Fruit Weight (g) ($0.047)$.

Days to first female flower had high negative direct effect $(-0.413)$ on yield.

Negative indirect effect were seen through vine length 90 days after sowing (-0.161), number of leaves 45 days after sowing ($0.030)$, days to first male flowering $(-0.024)$, node to first female flowering $(-0.078)$, days to 50 per cent flowering (-0.169), days to first harvest $(-0.383)$ and sex ratio $(-0.033)$.

Days to 50 per cent flowering had low negative direct effect $(-0.178)$ on yield.

Negative indirect effect were observed through vine length 90 days after sowing ($0.138)$, number of leaves 45 days after sowing $(-0.026)$, days to first female flowering ($0.492)$, days to first male flowering $(-0.010)$, node to first female flowering (-0.071), days to first harvest $(-0.402)$ and sex ratio (-0.035). Node to first male flower had low positive 
direct effect (0.119) on yield. Positive indirect effect was observed through vine length at 45 days after sowing (0.018), days to last harvest (0.145), number of fruits per plant (0.472), fruit yield per plot (0.348), average fruit weight (0.352), fruit length (0.018) and fruit diameter (0.055).

Node to first female flower had low negative direct effect $(-0.010)$ on yield.

Negative indirect effect were observed through vine length 90 days after sowing (0.115 ), days to first female flowering ($0.489)$, days to first male flowering (-0.012), days to 50 per cent flowering (-0.01), days to first harvest $(-0.405)$ and sex ratio $(-0.035)$.

Sex ratio had low negative direct effect (0.073 ) on yield. Negative indirect effect were observed through vine length 90 days after sowing (-0.123), days to first female flowering (-0.324), days to first male flowering (-0.056), node to first female flowering (-0.058), days to 50 per cent flowering (-0.124), days to first harvest ($0.312)$ and sex ratio $(-0.073)$.

Vine length at 90 days after sowing had direct negative effect $(-0.120)$ along with negative indirect effect through days to first female flowering (-0.224), days to first male flowering (-0.077), node to first female flowering (-0.043), days to 50 per cent flowering (-0.101), days to first harvest ($0.205)$ and sex ratio $(-0.056)$.

Days to first harvest had high negative direct effect (-0.375) on yield. Negative indirect effect were observed through vine length 90 days after sowing (-0.160), days to first female flowering $(-0.418)$, days to first male flowering (-0.014), node to first female flowering (-0.052), days to 50 per cent flowering (-0.151) and sex ratio (-0.039).
Days to last harvest had positive direct effect (0.160) on yield. Positive indirect effect was observed through node to first male flowering (0.194), number of fruits per plant (0.450), fruit yield per plot (0.271), average fruit weight (0.280), fruit length (0.011) and fruit diameter (0.057).

Number of fruits per plant had high positive direct effect (0.486) on yield. Positive indirect effect was observed through node to first male flowering (0.114), days to last harvest (0.176), fruit yield per plot (0.260), average fruit weight $(0.306)$, fruit length $(0.017)$ and fruit diameter (0.086).

Fruit yield per plot had high positive direct effect (0.293) on yield. Positive indirect effect was observed through node to first male flowering (0.176), days to last harvest (0.195), number of fruits per plant (0.490), average fruit weight (0.304), fruit length (0.012) and fruit diameter (0.103). Average fruit weight had high positive direct effect (0.318) on yield. Positive indirect effect was observed through node to first male flowering (0.106), days to last harvest (0.189), number of fruits per plant $(0.555)$, fruit yield per plot (0.297), fruit length (0.011) and fruit diameter (0.103).

Fruit length had low positive direct effect (0.012) on yield. Positive indirect effect was observed through node to first male flowering (0.155), days to last harvest (0.203), number of fruits per plant (0.355), fruit yield per plot $(0.430)$, average fruit weight $(0.0 .284)$ and fruit diameter (0.046).

Fruit diameter had low positive direct effect (0.140) on yield. Positive indirect effect was observed through node to first male flowering $(0.155)$, days to last harvest $(0.167)$, number of fruits per plant (0.337), fruit yield per plot (0.359), average fruit weight (0.292) and fruit length (0.014). 


\section{References}

Aykroyd W.R., 1963. The nutritive value of Indian foods and the planning of satisfactory diets. ICMR Special Report Series No. 42.

Chowdhury, D. and Sarma, K. C., 2002, Studies on variability, heritability, genetic advance and correlations in ridge gourd (Luffa acutangula Roxb.). Hort. J., 15(3): 53-58.

Johnson HW, Robinson HF, Comstock RE., 1955, Genotypic and phenotypic correlations in soybeans and their implication in selection. Agron $J$ 47: 477-483.

Khattra AS, Singh NJ, Thakur JC., 1994, Heterosis and correlation studies in bitter gourd. Veg Sci 21: 68-71.

Mangal JL, Dixit J, Padita ML, Sidhu AS., 1981, Genetic variability and correlation studies in bitter gourd. (Momordica Charantia L.). Indian J Hort 38: 94-99.

Panse, V. G. and Sukhatme, P. V., 1985, Statistical Methods for Agricultural Workers $\left(2{ }^{\text {nd }}\right.$ Edn. $)$, Indian Council of
Agricultural Research, New Delhi, $381 \mathrm{p}$.

Prasanna, S. C., Krishnappa, K. S. and Reddy, N. S., 2002, Correlation and path coefficient analysis studies in ridge gourd. Curr. Res., Univ. Agric. Sci., Bangalore, 31(9/10): 150-152.

Rajeswari KS., 1998, Genetic studies in bitter gourd (Momordica charantia L.) through diallel analysis. M.Sc. (Hort.) Thesis, Tamil Nadu Agriculture University.

Rao, B. N., Rao, P. V and Reddy, B. M. M., 2000, Correlation and path analysis in the segregating population of ridge gourd (Luffa acutangula (Roxb.) L.) Crop Res., 20 (2): 338-342.

Srivastava VK, Srivastava LC., 1976, Genetic parameters, correlation coefficients and path analysis in bitter gourd. Indian J. Hort., 33: 66-70.

Varalakshmi, B., Rao, P. V. and Reddy, Y. N., 1995, Genetic variability and heritability in ridge gourd (Luffa acutangula). Indian J. Agric. Sci., 65 (8): 608-610.

Wright, S., 1921. Correlation and causation. J. Agric. Res., 20: 557-587.

\section{How to cite this article:}

Ramesh, N.D., Praveen Choyal, Radhelal Dewangan, Pushpa S. Gudadinni, Priyanka P. Ligade and Kalyan Singh Seervi. 2018. Correlation and Path Analysis Study in Studied in Ridge Gourd (Luffa acutangula (L.) Roxb.). Int.J.Curr.Microbiol.App.Sci. 7(08): 1511-1519. doi: https://doi.org/10.20546/ijcmas.2018.708.172 